\title{
Language-Association Cortex Asymmetry in Autism and Specific Language Impairment
}

\author{
Lies De Fossé, MS, ${ }^{1,2}$ Steven M. Hodge, MA, ${ }^{1,2}$ Nikos Makris, MD, PhD, ${ }^{1}$ David N. Kennedy, PhD, ${ }^{1}$
}

Verne S. Caviness, Jr, MD, DPhil, ${ }^{1}$ Lauren McGrath, BS, ${ }^{3}$ Shelley Steele, BA, ${ }^{3}$ David A. Ziegler, BS, ${ }^{1}$

Martha R. Herbert, MD, PhD, ${ }^{1}$ Jean A. Frazier, MD, ${ }^{4,5}$ Helen Tager-Flusberg, $\mathrm{PhD},{ }^{3}$ and Gordon J. Harris, $\mathrm{PhD}^{2}$

\begin{abstract}
Language deficits are among the core impairments of autism. We previously reported asymmetry reversal of frontal language cortex in boys with autism. Specific language impairment (SLI) and autism share similar language deficits and may share genetic links. This study evaluated asymmetry of frontal language cortex in a new, independent sample of right-handed boys, including a new sample of boys with autism and a group of boys with SLI. The boys with autism were divided into those with language impairment (ALI) and those with normal language ability (ALN). Subjects (righthanded, aged 6.2-13.4 years) included 22 boys with autism (16 ALI and 6 ALN), 9 boys with a history of or present SLI, and 11 normal controls. MRI brain scans were segmented into grey and white matter; then the cerebral cortex was parcellated into 48 gyral-based divisions per hemisphere. Group differences in volumetric asymmetry were predicted $a$ priori in language-related regions in inferior lateral frontal (Broca's area) and posterior superior temporal cortex. Language impaired boys with autism and SLI both had significant reversal of asymmetry in frontal language-related cortex; larger on the right side in both groups of language impaired boys and larger on the left in both unimpaired language groups, strengthening a phenotypic link between ALI and SLI. Thus, we replicated the observation of reversed asymmetry in frontal language cortex reported previously in an independent autism sample, and observed similar reversal in boys with SLI, further strengthening a phenotypic link between SLI and a subgroup of autism. Linguistically unimpaired boys with autism had similar asymmetry compared with the control group, suggesting that Broca's area asymmetry reversal is related more to language impairment than specifically to autism diagnosis.
\end{abstract}

Ann Neurol 2004;56:757-766

Autism is a developmental disorder defined by impairments in social interaction and communication skills as well as repetitive behaviors and stereotyped interests. ${ }^{1}$ Deficits in language functioning are a commonly observed aspect of the impairments in communication skills. These difficulties may range from no functional language, to impairments in phonological processing, vocabulary, and higher order syntax and semantics, relative to age-matched peers. ${ }^{2-4}$ There are, however, some children with autism who have normal language skills. ${ }^{5}$ In a large-scale study of language in a heterogeneous group of children with autism, the subgroup of autistic children with impaired language skills were shown to have a similar profile of language impairment to children with specific language impairment (SLI). ${ }^{6}$

SLI is a disorder characterized by delayed language development in the absence of other cognitive impairments. Both autism and SLI have strong genetic bases (for reviews, see Fisher and colleagues ${ }^{7}$ as well as San- tangelo and Folstein ${ }^{8}$ ). Family and twin studies found that first-degree relatives of probands with autism more frequently display deficits in language skills than the occurrence rate in the general population. ${ }^{9-11}$ Furthermore, siblings of children with SLI have an elevated risk for autism. ${ }^{12}$ Genetic linkage studies in these two developmental disorders point to overlapping regions on chromosome $7 \mathrm{q}^{13-15}$ and chromosome $13 \mathrm{q} \cdot{ }^{14,16}$ In genetic studies of autism, signals on both $7 \mathrm{q}$ and $13 \mathrm{q}$ increase significantly when linkage studies restrict their analyses to autism families with clear signs of language impairment. ${ }^{17,18}$ These findings suggest that the genetic abnormalities that lead to the phenotype of developmental language disorders ${ }^{7}$ may overlap with the genetic alterations that are liability factors for autism.

Anatomical asymmetries in language-related cortex have been associated with language laterality dominance from Wada tests ${ }^{19-21}$ and by dichotic listening tests. ${ }^{22}$ Autopsy and magnetic resonance imaging (MRI) studies
From the ${ }^{1}$ Center for Morphometric Analysis and ${ }^{2}$ Radiology Computer Aided Diagnostics Laboratory, Massachusetts General Hospital; ${ }^{3}$ Boston University School of Medicine; ${ }^{4}$ Department of Psychiatry, Harvard Medical School, Boston; and ${ }^{5}$ Child Psychiatry Outpatient Clinic, McLean Hospital, Belmont, MA.

Received Feb 12, 2004, and in revised form Jul 23. Accepted for publication Jul 23, 2004.
Published online Oct 11, 2004 in Wiley InterScience (www.interscience.wiley.com). DOI: 10.1002/ana.20275

Address correspondence to Dr Harris, RAD CADx LAB, MGH, Zero Emerson Place \#3A, Boston, MA 02114.

E-mail: gjharris@partners.org 
have shown that the language region in the inferior frontal cortex (Broca's area) is larger in the left hemisphere than in the right in most right-handed, typically developed individuals. ${ }^{19,23-29}$ In contrast, studies of people with developmental language disorders have found reduced or reversed asymmetry patterns in these areas. For example, the pars triangularis of the inferior frontal cortex is smaller in the left hemisphere of children with SLI relative to control subjects. ${ }^{30}$ The KE family includes a three-generation pedigree with a dominantly inherited speech disorder. ${ }^{31}$ Voxel-based morphometry (VBM) indicated that members of the KE family affected with a speech and language disorder that is associated with a specific mutation in the FOXP2 gene on chromosome $7 \mathrm{q}^{31}$ had less gray matter in left inferior frontal cortex (Brodmann area 9/44) as compared with the unaffected family members. ${ }^{32,33}$ The inferior frontal gyrus in adults with developmental language disorders often presented with an extra sulcus compared with a normal control group. ${ }^{34}$

Some investigators also have reported abnormalities in language regions, including Broca's area, in autism. A decrease of gray matter density in Brodmann area 45 in the left inferior frontal gyrus in adults with autism was reported using VBM. ${ }^{35}$ Rightward volumetric asymmetry of inferior frontal cortex pars opercularis was observed in right-handed 7- to 11-year-old children with autism, in contrast with leftward asymmetry in a right-handed control group. ${ }^{36}$

Studies of the planum temporale (PT) in subjects with autism and SLI have yielded more varied results. The PT, including the ascending part bordered by the posterior ascending ramus, was larger in the right hemisphere in children with SLI and larger on the left in normal control subjects. ${ }^{30}$ In the same study, the PT without the ascending part showed less leftward asymmetry in children with SLI compared with control subjects, but the difference was not significant. Reduced left PT volume was reported in adults with autism when compared with normal adults. ${ }^{37}$ A contrasting result was reported in a study of children with autism that focused on volumetric symmetry ${ }^{36}$ : the PT had more extreme leftward asymmetry (ie, larger volume in the left hemisphere) in children with autism compared with a normal control group. Other studies also suggest posterior superior temporal abnormalities in autism and SLI. Boys with SLI have atypical perisylvian asymmetries due to a larger right perisylvian area compared with normal controls. ${ }^{38}$ Finally, a recent study reported abnormalities in the superior temporal gyrus in a majority of autistic children. ${ }^{39}$

Motivated by the possible genetic linkage between autism and SLI and the similarity in language profile described above, this study directly compares asymmetry patterns in cortical regions related to language processing between groups of autistic boys with normal and impaired language skills, a group of boys with SLI, and a group of normal control boys.

We postulated that the autism group with impaired language skills would show similar cortical languagerelated abnormalities as the group with SLI. We identified, based on earlier findings by our research group, ${ }^{36,40,41}$ several regions of interest in this study. These areas, related to language processing, are in lateral inferior frontal cortex: pars triangularis and opercularis of the inferior frontal gyrus and the frontal operculum (Broca's area); and in temporoparietal cortex: anterior and posterior superior temporal gyrus, the planum temporale, anterior and posterior supramarginal gyrus, angular gyrus and the parietal operculum (broader Wernicke's area).

We hypothesized that the right-handed languageimpaired boys with autism and the boys with SLI both would show a reversal of volumetric asymmetry (larger in right hemisphere) in inferior frontal gyrus (referred to as "F3" in the neuroanatomical parcellation system described below), involving Broca's area, in contrast with both the normal control group and the boys with autism who had normal language skills (larger in left hemisphere).

We also hypothesized that the exaggerated leftward asymmetry of the PT found previously in children with autism ${ }^{36}$ would be replicated in a new sample, because the same methods were used in both studies. This could clarify whether the inconsistency between this finding and others, as mentioned above, is an issue of methodology. In addition, we tested whether this exaggerated leftward asymmetry would be related to language impairment.

Finally, we examined whether there is a difference between groups in the pattern of volumetric asymmetry in the regions surrounding F3 and PT, for example, frontal operculum (FO) and regions making up the broader Wernicke's area: superior temporal gyrus (T1), a combined region consisting of supramarginal and angular gyrus (SG+AG), and the parietal operculum (PO). These secondary language-related regions serve as a test of specificity of the asymmetry abnormalities to F3 and/or PT, because we did not observe abnormalities in these bordering regions previously and hypothesized that these regions would not differentiate groups, whereas F3 would differ based on language impairment.

\section{Subjects and Methods}

Subjects

The children with autism and SLI were enrolled as part of a program project on language and related social cognitive functioning in autism. All subjects were male and all were predominantly right-handed, as determined by the Edinburgh Inventory in NC subjects, ${ }^{42}$ and by the Dean Laterality Preference Schedule in all other groups. ${ }^{43,44}$ Control subjects were part of an ongoing neuroimaging study of childhood-onset psychiatric illnesses. The study included four groups of subjects: boys with autism with either impaired language (ALI; $\mathrm{n}=16$ ) or 
normal language skills (ALN; $\mathrm{n}=6$ ), boys with diagnosis of the presence or history of SLI $(n=9)$, and typically developing control boys $(\mathrm{NC} ; \mathrm{n}=11)$. The age range of the four groups was 6.2 to 13.4 years (Table 1). There were no significant differences in age among the groups. Subjects were excluded if they had frank neurological damage or had been diagnosed with fragile X, neurofibromatosis, cerebral palsy, tuberous sclerosis, William's syndrome, or Down's syndrome. The control group had no DSM-IV axis I diagnosis on structured and clinical interviews. ${ }^{1}$

Full-scale, verbal, and nonverbal intelligence quotients (IQs) ${ }^{45,46}$ of the four groups are shown in Table 1. There were significant differences in full-scale IQ between the groups $(\mathrm{F}[3,38]=13.4, p<0.0001)$ with the languageimpaired groups (ALI and SLI) having significantly lower verbal $(\mathrm{t}[38]=4.4, p<0.0001)$ and nonverbal IQ $(\mathrm{t}[38]=$ $4.2, p<0.001$ ) than the unimpaired language groups (ALN and NC). The ALI group had significantly lower verbal IQ than the SLI group $(\mathrm{t}[38]=2.6, p=0.01)$, but there was no significant difference in nonverbal IQ $(\mathrm{t}[38]=1.2, p=$ 0.2 . The same pattern was observed with the ALN group who had significantly lower verbal IQ than the NC group, but comparable nonverbal IQ (verbal: $\mathrm{t}[38]=2.3, p=$ 0.03 ; nonverbal: $\mathrm{t}[38]=0.7, p=0.5)$.

\section{Autism Testing}

A diagnosis of autism was established on the basis of the Autism Diagnostic Interview-Revised ${ }^{47}$ and the Autism Diagnostic Observation Schedule. ${ }^{48}$ In addition, an expert clinician examined all participants to confirm that they met DSM-IV criteria for autism. ${ }^{1}$

\section{Language Testing}

Language skills were tested using the Clinical Evaluation of Language Fundamentals (CELF, 3rd ed $)^{49}$ and the nonsense word repetition subtest of the NEPSY..$^{50}$ The CELFIII is an omnibus test of language ability, measuring higher order syntax and semantics. There are six subtests, three in the receptive domain and three in the expressive domain, and these are combined to form expressive and receptive composite scores and a total language standard score (each with a mean of 100 and a standard deviation of 15). Criteria for language impairment among the boys with autism were a CELF score of 81 or lower, or a score 6 or lower on the nonsense word repetition subtest. All the boys included in the SLI group had a history of significant language delay and had been clinically referred for treatment. Of the nine boys in this group, five had current language impairment according to the inclusion criteria mentioned above. The four boys with only a history of language impairment fell within the normal limits on the language measures at the time of testing; however, this is not uncommon in SLI. ${ }^{51}$ As noted, all the boys with autism in the ALI group met the criteria for current language impairment described here. The boys with autism in the ALN group all scored within the normal range on the CELF and the nonsense word repetition task. Although the control group did not undergo the full battery of testing of language skills, all NC subjects had normal verbal IQ and reading scores, no obvious language abnormality, and none had any history of language delay or language-based learning disabilities.

\section{Image Acquisition}

Subjects were trained in a mock scanner before actual MR scanning. ${ }^{52}$ This allowed us to acquire high-resolution structural scans using behavioral shaping techniques to minimize movement without sedating the subjects. All images were acquired on a General Electric (Milwaukee, WI) 1.5T Signa MRI system. During the course of the study, there was a software upgrade on the scanner. In addition, a protocol was implemented in which two faster anatomical series were acquired to maximize the chance of getting a series without motion artifacts (we refer to this as the dual acquisition protocol). By taking two more rapid acquisitions, we were more likely to obtain at least one that was free of motion artifact. Validation studies were performed before and after the upgrade and the transition from a single series acquisition to the shorter dual acquisition protocol.

A single series of coronal three-dimensional T1-weighted MR scans was acquired from five normal control subjects with the following parameters: TR, 13.8 milliseconds; TE, 2.8 milliseconds; TI, 300 milliseconds; flip angle, 25 degrees; slice thickness, $1.5 \mathrm{~mm}$ (contiguous); image matrix, $256 \times 256$ pixels; inplane pixel dimension, $0.9375 \times 0.9375 \mathrm{~mm}^{2}$; FOV, $240 \mathrm{~mm}$.

The dual acquisition protocol was used for 14 scans of subjects with autism and had the following parameters

Table 1. Characteristics of the Subject Groups (mean $\pm S D$ )

\begin{tabular}{|c|c|c|c|c|}
\hline Characteristic & $\mathrm{NC}(\mathrm{n}=11)$ & $\operatorname{ALN}(\mathrm{n}=6)$ & $\operatorname{ALI}(\mathrm{n}=16)$ & SLI $(n=9)$ \\
\hline Age $(\mathrm{yr})$ & $10.4 \pm 2.7$ & $8.3 \pm 0.9$ & $9.8 \pm 2.1$ & $9.9 \pm 2.3$ \\
\hline Full-scale IQ ${ }^{a}$ & $114.5 \pm 11.3$ & $109.3 \pm 24.1$ & $78.3 \pm 14.7$ & $93.4 \pm 15.5$ \\
\hline Verbal IQ & $115.9 \pm 12.4$ & $97.7 \pm 19.3$ & $75.1 \pm 15.9$ & $92.5 \pm 15.6$ \\
\hline Nonverbal IQ & $110.6 \pm 11$ & $116.3 \pm 24.9$ & $87.9 \pm 14.3$ & $95.7 \pm 14.6$ \\
\hline \multicolumn{5}{|l|}{ Language testing } \\
\hline Nonword repetition ${ }^{\mathrm{b}}$ & - & $8.5 \pm 1.9$ & $6 \pm 2.3$ & $8.1 \pm 2.9$ \\
\hline CELF $^{c}$ & - & $101 \pm 12.1$ & $65.5 \pm 9.4$ & $85.4 \pm 11.2$ \\
\hline
\end{tabular}

${ }^{\text {aSLI }}$ and subjects with autism: Differential Abilities Scale ${ }^{45}$; control subjects: WISC-III. ${ }^{46}$

${ }^{\mathrm{b}}$ Repetition on Nonsense Words subtest of NEPSY. ${ }^{50}$

${ }^{\mathrm{c}}$ Clinical Evaluation of Language Fundamentals. ${ }^{49}$

$\mathrm{SD}=$ standard deviation; $\mathrm{NC}=$ normal control; ALN $=$ autism with normal language; ALI $=$ autism with language impairment; SLI $=$ specific language impairment. 
changed from those reported above: TR, 13.7 milliseconds; TE, 2.7 milliseconds; in-plane pixel dimension, $1.172 \times$ $1.172 \mathrm{~mm}^{2}$; FOV, $300 \mathrm{~mm}$. All other parameters were the same as reported above.

After a scanner software upgrade, six normal control subjects, eight subjects with autism, and nine subjects with SLI were scanned with the dual acquisition protocol. The following scanning parameters underwent minimal changes from the dual protocol listed above: TR, 11 milliseconds; TE, 1.90 milliseconds (FOV, 300mm). All other parameters were the same as reported above.

Validation studies were performed to determine the effect of the change in scanning protocol and software upgrade. Four people unrelated to the study agreed to be scanned with both the single and dual protocols. Three additional people volunteered to be scanned before and after the upgrade of the scanning software. We obtained the volumes of the right and left cerebral cortex for all scans. ${ }^{53,54}$ Not only is the cortex the basis for the subsequent parcellation and thus includes all the regions of interest in this study, but it also represents two intensity class boundaries (gray matter-extracerebral cerebrospinal fluid and gray matter-white matter) that would be expected to show any global effects of scan parameter changes. Intraclass correlation coefficients (ICCs) were calculated for consistency of the volume measurements. ${ }^{55,56}$ For the protocol change, the ICC for volume consistency was 0.936 , and for the scanning software upgrade the ICC was 0.944 . For both the scan protocol change and the upgrade, measurements of the cerebral cortex differed by less than $2 \%$ on average across the changes. Furthermore, because the measures of interest and hypotheses in this study involve cortical asymmetry measures, these changes in scan protocols would not be expected to have an impact on one hemisphere versus the other, and impact on asymmetry would be unlikely.

The dual protocol acquisitions of each subject were coregistered and averaged. ${ }^{57,58}$ This averaged series was compared with both separate acquisitions and the version with the least motion artifact was chosen for analysis. The selected series of each subject was reoriented in a common stereotactic space, ${ }^{59,60}$ but without rescaling the image parameters. The images then were resliced in the position normalized coronal plane with $1.5 \mathrm{~mm}$ slice thickness. The images were magnified to an in-plane pixel resolution of $0.837 \mathrm{~mm}$, except for those series where the field of view was $240 \mathrm{~mm}$. A correction was performed to remove intensity nonuniformity effects. ${ }^{61,62}$

\section{Morphometric Analysis of Images}

The cerebral cortex was extracted from the image as part of a whole-brain segmentation, which used semiautomated procedures based on intensity contour mapping and differential intensity contour algorithms that have been described previously. ${ }^{53,54}$ After segmentation, the neocortex was divided into 48 parcellation units (PUs) per hemisphere using a selfreferential system based on the gyral pattern of the cortex. $^{63,64}$ The anterior-posterior borders of the PUs are defined by a set of coronal planes according to anatomical landmarks. The medial-lateral and dorsal-ventral borders of the PUs are defined by identifying the neocortical fissures. This method is extensively described in Rademacher and col- leauges ${ }^{63}$ and Caviness and colleagues. ${ }^{64}$ See Figure 1 for a schematic of the parcellation units of interest in this study and Table 2 for the definitions of the parcellation unit borders. The whole-brain segmentation and parcellation of the cortex was performed by a technician extensively trained in neuroanatomy and supervised by a neuroanatomist. Both were blind to the group affiliation of the subjects.

Some of the regions separated by a single boundary as described in the method in Caviness and colleauges ${ }^{64}$ and studied in Herbert and colleauges ${ }^{36}$ are combined in this article. We did this to decrease the variability in the volume of these regions and limit the number of comparisons. For example, there is substantial fluctuation in the volume of the AG and the SG, in part because the intermediary sulcus of Jensen, which forms the posterior border of SG and the anterior border of AG, is highly variable. ${ }^{64}$ Therefore, AG was combined with the anterior and posterior portion of SG, into SG+AG. For similar reasons, inferior frontal gyrus, pars opercularis (F3o), and pars triangularis (F3t) were combined into F3, and the anterior and posterior portions of the superior temporal gyrus (T1a and T1p) were combined into T1.

The volumetric asymmetry of a PU is estimated by the symmetry index ${ }^{24}$

$$
\text { symmetry index }=100 \times \frac{\text { Left }_{\text {.volume }}-\text { Right }_{\text {.volume }}}{\frac{1}{2}\left(\text { Left }_{\text {volume }}+\text { Right }_{\text {.volume }}\right)}
$$

where left PU volume and right PU volume are identically labeled PUs in the left and right hemisphere. Positive values indicate that the PU is larger in the left hemisphere. Thus, if the symmetry index is 20 , the volume in the left hemisphere is larger than the right hemisphere by $20 \%$ of the average volume of the left and right hemispheres. The symmetry index is a ratio of volumes, and thus, a valueless quanitity. We examined the impact of cerebrum size on the analyses of regional asymmetry.

\section{Data Analysis}

First, one-way analyses of variance (ANOVAs) with pooled estimates of error variance were used to test for betweengroup differences in the raw volumes of the whole cerebrum, neocortex, and (by hemisphere) the primary parcellation units of interest: inferior frontal gyrus (F3) and the planum temporale (PT). We used Tukey-Kramer Honestly Significant Difference (HSD) tests for post hoc pairwise comparisons because of the conservative nature of the test when used with unequal sample sizes. ${ }^{65}$ Based on our primary hypotheses driven by our prior findings, we next tested differences in the symmetry index of F3 and PT, separately, with oneway ANOVAs with Group (ALI, ALN, SLI, NC) as the between subjects variable. Planned linear contrasts were used to determine (1) whether the language-impaired groups (SLI and ALI) differed from language unimpaired groups (ALN and NC), and (2) whether subjects with autism differed from their language cogroup (ALI vs SLI; ALN vs NC). Then, a multivariate ANOVA was used to test group differences in the symmetry index of parcellation units that border F3 and PT: FO, T1, SG+AG, and the PO. Symmetry indices were 


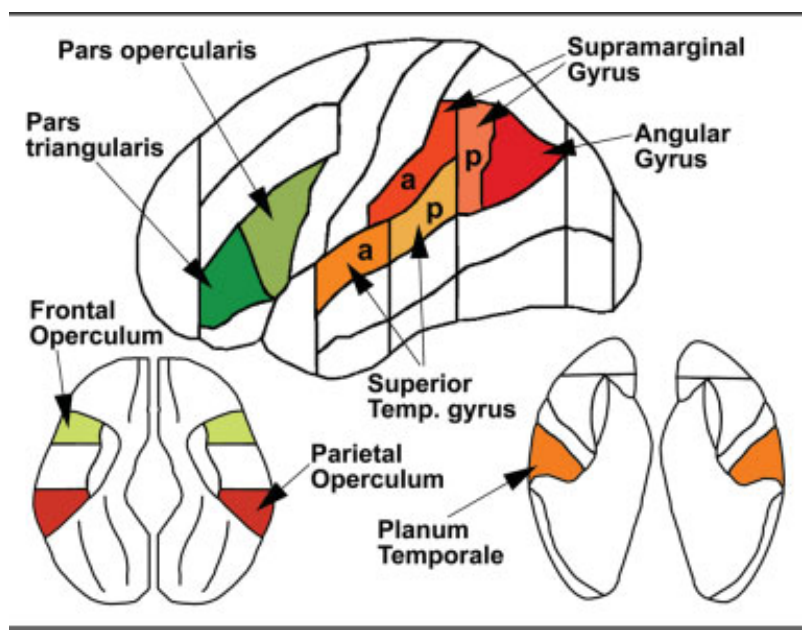

Fig 1. Cortical parcellation units and their location. The top image is a representation of the lateral surface of the brain. Inferior frontal gyrus, pars opercularis plus pars triangularis are combined to form "F3" (Broca's area). The lower left and right images represent the upper and lower surface of the Sylvian fissure, respectively. $a=$ anterior; $p=$ posterior.

further analyzed within each group using two-tailed $t$ tests to assess whether individual regions differed significantly from zero (ie, whether regions were significantly asymmetric within group). Correlation analyses were performed relating principal regions of interest, cerebral cortex and cerebrum volume, and IQ scores. All analyses were performed using the statistical software package JMP 5.0.1.2 (SAS Institute, Cary, NC).

\section{Results}

Mean volumes (in $\mathrm{cm}^{3}$ ) for the whole cerebrum, cerebral cortex, and parcellation units are given in Table 3 . There is a trend toward difference in cerebrum volume among the groups $(\mathrm{F}[3,38]=2.66, p=0.06)$, where the ALI and ALN groups have greater cerebral volume than the NC and SLI groups. There is a $106 \mathrm{~cm}^{3}$ mean volume difference between the SLI and ALI groups (means are 1,167 and $1,273 \mathrm{~cm}^{3}$, respectively, $p=$ $0.04)$. A similar group effect is found with cerebral cortex $(\mathrm{F}[3,38]=2.86, p=0.05)$, where the post hoc significant difference is again between the SLI $\left(699 \mathrm{~cm}^{3}\right)$ and ALI $\left(767 \mathrm{~cm}^{3}\right)$ groups $(p=0.03)$ There were no significant group differences in the raw volume of F3 or PT by hemisphere (left F3: F[3,38] = 1.8, $p=0.2$; right $\mathrm{F} 3: \mathrm{F}[3,38]=1.1, p=0.4$; left PT: $\mathrm{F}[3,38]=0.5, p=0.7$; right PT: $\mathrm{F}[3,38]=0.6$, $p=0.6)$.

Mean symmetry indices for the cerebral cortex and parcellation units are given in Table 4. Cerebral cortex as a whole was highly symmetric, with less than $0.5 \%$ mean asymmetry in all groups and no between-group differences. Two-tailed $t$ tests within-group (onesample) of symmetry indices indicated that F3 in the ALI group was significantly asymmetric, larger on the right than the left side $(\mathrm{t}[15]=-2.7, p=0.017)$. PT was significantly asymmetric, larger on the left side, in both the ALI $(\mathrm{t}[15]=4.4, p=0.0006)$ and SLI $(\mathrm{t}[8]$ $=5.0, p=0.001)$ groups, but not in $\mathrm{ALN}(\mathrm{t}[5]=$ $1.1, p=0.30)$ or $\mathrm{NC}(\mathrm{t}[10]=1.7, p=0.12)$. ALI

Table 2. Definitions of Regions of Interest Based on Cerebral Cortex Parcellation Units ${ }^{63,64}$

Borders

Parcellation Unit

$\begin{array}{lll}\text { Anterior } \quad \text { Posterior } & \text { Ventral }\end{array}$

Lateral surface

$\mathrm{F} 3 \mathrm{t}$

F3o

SGa

SGp

AG

T1a

T1p

Intrasylvian surface

$\mathrm{FO}$

$\mathrm{PO}$

PT
Inferior frontal gyrus, pars triangularis

Inferior frontal gyrus, pars opercularis

Supramarginal gyrus, anterior portion

Supramarginal gyrus, posterior portion

Angular gyrus

Superior temporal gyrus, anterior portion

Superior temporal gyrus, posterior portion

Frontal operculum

Parietal operculum

Planum temporale

$\begin{array}{llll}\text { plane A } & \text { aar } & \text { ahr } & \text { if } \\ \text { aar-if } & \text { prc-plane O } & \text { ahr-prc } & \text { if } \\ \text { plane P } & \text { plane D } & \text { phr } & \text { poc/ip } \\ \text { plane D } & \text { ij-plane E } & \text { st } & \text { ip } \\ \text { ij-plane E } & \text { plane F } & \text { st/lo } & \text { ip } \\ \text { plane B } & \text { plane C } & \text { st } & \text { Syl-HM } \\ \text { plane C } & \text { plane D } & \text { st } & \text { Syl-HM } \\ & & & \\ \text { INS-ANT } & \text { plane O } & \text { cir } & \text { Syl-HM } \\ \text { plane P } & \text { plane D } & \text { cir - syl } & \text { Syl-HM } \\ \text { He-ant } & \text { plane D } & \text { He/cir/Syl } & \text { Syl-HM }\end{array}$

aar $=$ anterior ascending rhamus of the Sylvian fissure; aar-if $=$ most anterior point of the anterior ascending rhamus of the Sylvian fissure (where it intersects or is close to the inferior frontal sulcus; $a h r=$ anterior horizontal rhamus of the Sylvian fissure; cir = circular sulcus of the insula; $\mathrm{He}=$ Heschl's sulcus; He-ant = anterior limit of the Heschl's gyrus; if = inferior frontal sulcus; ij = intermediate sulcus of Jensen; INS-ANT = anterior limit of the insular cortex; ip = intraparietal sulcus; lo = lateral occipital sulcus; phrs = posterior horizontal rhamus of the Sylvian fissure; plane A = most anterior point of the anterior horizontal rhamus of the Sylvian fissure; plane B = isthmus of the temporal and frontal lobes; plane $\mathrm{C}=$ anterior limit of the first transverse sulcus of Heschls; plane $\mathrm{D}=$ posterior limit of the Sylvian fissure; plane $\mathrm{E}=$ inferior limit of the intermediate sulcus of Jenson; plane $\mathrm{F}=$ operculization of the Sylvian fissure; plane $\mathrm{O}=$ point where the precentral sulcus intersects the Sylvian fissure; plane $\mathrm{P}=$ point where the postcentral sulcus intersects the Sylvian fissure; poc $=$ postcentral sulcus; prc $=$ precentral sulcus; st $=$ superior temporal sulcus; Syl-HM = lip of the Sylvian fissure th the hemispheric margin; Syl = sylvian fissure. 
also showed significant rightward asymmetry in $\mathrm{SG}+\mathrm{AG}(\mathrm{t}[15]=-3.2, p=0.006)$ and leftward asymmetry in PO $(\mathrm{t}[15]=3.1, p=0.008)$.

Both language-impaired groups (SLI and ALI) showed a mean rightward Broca's area asymmetry in inferior frontal gyrus (F3), which was a reversal of the mean leftward asymmetry seen in the unimpaired language groups (ALN and NC, see Table 4 and Fig 2). ANOVA analysis showed this difference to be significant $(\mathrm{F}[3,38]=2.9, p=0.05)$ and the group contrast showed that the difference was along the language dimension (SLI and ALI vs ALN and NC: $\mathrm{t}[38]=2.8$, $p=0.007)$. There were no differences between SLI and ALI $(\mathrm{t}[38]=0.5, p=0.6)$ or ALN and NC $(\mathrm{t}[38]=0.1, p=0.9)$. Because there were significant between-group differences in cerebral volume, we added this variable to the analysis of F3 group differences, which remained significantly after accounting for cerebrum size $(\mathrm{F}[3,1,37]=3.3, p=0.03)$.

The asymmetry of the PT was significantly leftward biased in both the SLI and ALI groups, but the leftward asymmetry was not significant in the ALN and NC groups (see analyses above), which suggests exaggerated asymmetry in the language-impaired groups (see Table 4). However, the between-group comparison was not significant in the overall ANOVA $(\mathrm{F}[3,38]=$ $0.7, p=0.5$ ) or in the language group contrast (SLI and ALI vs ALN and NC: $\mathrm{t}[38]=1.4, p=0.17)$. There were no differences between SLI and ALI ( $\mathrm{t}[38]$ $=0.1, p=0.9)$, or ALN and $\mathrm{NC}(\mathrm{t}[38]=0.1, p=$ $0.9)$.

A one-way multivariate ANOVA was performed to assess group differences among broader language areas that border on F3 and PT: FO, T1, SG+AG, and PO. Wilks' $\Lambda$ multivariate test did not show any significant differences in the overall asymmetry for these regions $(\mathrm{F}[12,92.9]=1.6, p=0.11)$. Furthermore, post hoc univariate ANOVAs for each dependent variable showed no significant group differences.

There was a significant correlation between verbal
IQ and F3 asymmetry $(\mathrm{r}[39]=0.43, p=0.04)$, with higher verbal IQ associated with more leftward F3 asymmetry. The correlation analysis between F3 and nonverbal IQ was not significant. Cerebral cortex and total cerebral volume were not significantly associated with IQ scores (verbal, nonverbal, and full-scale IQ all had correlations with cerebral cortex and total cerebrum volume with $p>0.30$ ).

\section{Discussion}

This study investigated whether reversal of normal leftright volumetric asymmetry patterns in frontal cortical language regions (Broca's area) is more closely related to language impairment than to autism diagnosis. This study compared boys with autism who had either normal or impaired language skills to control groups that included developmentally normal boys and boys with SLI. The results suggest that the observed abnormal asymmetry in language-related brain regions in SLI and ALI was more closely related to language impairment than to autism diagnosis. The boys with autism who had normal language skills were similar to the normal control boys in leftward volumetric asymmetry of inferior frontal gyrus, pars opercularis plus pars triangularis (F3). In contrast, the boys with autism who had impaired language skills showed reversed (rightward) asymmetry in F3, as did the boys with SLI. Boys with language impairment (SLI and ALI) had lower verbal IQ scores than language-normal subjects (ALN and NC), as expected. The asymmetry of F3 frontal language-association cortex was correlated with verbal IQ scores, but not with nonverbal IQ, further supporting the relationship between Broca's area asymmetry and language abilities in right-handed subjects.

Although this study, to our knowledge, is the first to directly assess the relationship between autism and language-impairment domains with Broca's area asymmetry patterns, several reports have observed reversed Broca's asymmetry in either SLI or autism. This study replicated and expanded on a previous study in an in-

Table 3. Volumes (mean \pm SD) of Brain Regions of Interest ( $\mathrm{ml}$ )

\begin{tabular}{|c|c|c|c|c|c|c|c|c|}
\hline \multirow[b]{2}{*}{ Brain Region } & \multicolumn{2}{|c|}{$\mathrm{NC}$} & \multicolumn{2}{|c|}{ ALN } & \multicolumn{2}{|c|}{ ALI } & \multicolumn{2}{|c|}{ SLI } \\
\hline & Right & Left & Right & Left & Right & Left & Right & Left \\
\hline Whole cerebrum & $609.9 \pm 27.6$ & $611.1 \pm 30.3$ & $622.4 \pm 35.2$ & $621.6 \pm 35.5$ & $636.3 \pm 62.1$ & $636.9 \pm 61.4$ & $584.9 \pm 35.6$ & $582.0 \pm 31.7$ \\
\hline Whole cerebral cortex & $367.1 \pm 16.9$ & $368.2 \pm 20.1$ & $375.1 \pm 25.9$ & $375.8 \pm 32.2$ & $383.7 \pm 37.0$ & $383.7 \pm 37.3$ & $349.1 \pm 20.0$ & $349.9 \pm 18.9$ \\
\hline \multicolumn{9}{|c|}{ Primary parcellation units of interest } \\
\hline Broca's Area (F3) & $8.6 \pm 1.5$ & $9.6 \pm 2.2$ & $9.0 \pm 2.1$ & $9.7 \pm 1.7$ & $10.2 \pm 3.0$ & $8.7 \pm 1.9$ & $9.8 \pm 2.5$ & $7.9 \pm 1.7$ \\
\hline Planum temporale & $3.3 \pm 0.8$ & $3.9 \pm 0.9$ & $3.2 \pm 0.8$ & $3.6 \pm 0.5$ & $3.1 \pm 0.7$ & $4.1 \pm 1.0$ & $2.9 \pm 0.4$ & $3.9 \pm 0.8$ \\
\hline \multicolumn{9}{|c|}{ Bordering parcellation units of interest } \\
\hline Frontal operculum & $2.8 \pm 0.7$ & $2.6 \pm 0.7$ & $2.7 \pm 0.7$ & $2.4 \pm 0.7$ & $2.6 \pm 0.7$ & $2.7 \pm 0.7$ & $2.9 \pm 0.7$ & $2.3 \pm 0.9$ \\
\hline Superior temporal gyrus & $6.8 \pm 1.5$ & $6.7 \pm 1.0$ & $7.3 \pm 1.1$ & $6.7 \pm 1.3$ & $7.4 \pm 1.3$ & $6.9 \pm 1.4$ & $6.2 \pm 1.0$ & $6.3 \pm 0.9$ \\
\hline Supramarginal/angular & $18.4 \pm 3.8$ & $17.5 \pm 4.2$ & $18.5 \pm 5.4$ & $18.1 \pm 4.4$ & $21.3 \pm 2.9$ & $18.1 \pm 4.0$ & $15.4 \pm 2.8$ & $14.9 \pm 3.5$ \\
\hline Parietal operculum & $3.1 \pm 0.9$ & $3.7 \pm 0.9$ & $3.4 \pm 0.9$ & $3.3 \pm 0.9$ & $3.1 \pm 0.6$ & $4.2 \pm 1.3$ & $3.2 \pm 0.5$ & $3.4 \pm 0.9$ \\
\hline
\end{tabular}

Volumetric data for the parcellation units are provided as a reference for their symmetry indices, which are shown in Table 4.

$\mathrm{NC}=$ normal control; ALN = autism with normal language; ALI = autism with language impairment; SLI $=$ specific language impairment. 
Table 4. Symmetry Indices (mean \pm SD) for Lateral Inferior Frontal and Temporoparietal Language-Related Cortical Regions, and for the Entire Cerebral Cortex

\begin{tabular}{lrrrr}
\hline Brain Region & \multicolumn{1}{c}{ NC } & ALN & ALI & SLI \\
\hline Cerebral cortex & $0.3 \pm 1.9$ & $0.1 \pm 2.1$ & $-0.002 \pm 2.3$ & $0.3 \pm 1.6$ \\
Primary parcellation units of interest & & & & \\
$\quad$ Broca's area (F3) & $9.7 \pm 23.3$ & $8.2 \pm 32.5$ & $-14.2 \pm 21.1$ & $-20.3 \pm 39.8$ \\
$\quad$ Planum temporale (PT) & $15.7 \pm 30.8$ & $14.0 \pm 30.0$ & $27.2 \pm 24.9$ & $26.5 \pm 16.0$ \\
Bordering parcellation units of interest & & & $3.8 \pm 29.8$ & $-27.8 \pm 38.5$ \\
$\quad$ Frontal operculum & $-7.0 \pm 24.4$ & $-14.0 \pm 36.7$ & $-7.4 \pm 16.2$ & $2.5 \pm 18.3$ \\
$\quad$ Superior temporal gyrus & $0.7 \pm 27.0$ & $-10.1 \pm 25.8$ & $-18.0 \pm 22.5$ & $-5.4 \pm 22.9$ \\
$\quad$ Supramarginal and angular gyrus & $-5.6 \pm 25.0$ & $-1.1 \pm 25.0$ & $25.6 \pm 33.4$ & $4.0 \pm 26.0$ \\
$\quad$ Parietal operculum & $19.1 \pm 39.8$ & $-1.7 \pm 27.1$ & & \\
\hline
\end{tabular}

A positive value indicates the volume of the parcellation unit is larger in the left hemisphere, a negative value indicates larger volume in the right hemisphere.

${ }^{\text {aS }}$ ymmetry index $=100^{*}$ (left volume-right volume) $/[($ left volume + right volume $) / 2]$.

bLanguage-impaired groups (ALI, SLI) had mean rightward asymmetry, whereas the groups with normal language skills (NC, ALN) had mean leftward asymmetry (post hoc group contrast: $\mathrm{t}(38)=2.8, p=0.007$ ).

$\mathrm{SD}=$ standard deviation; $\mathrm{NC}=$ normal control; $\mathrm{ALN}=$ autism with normal language; $\mathrm{ALI}=$ autism with language impairment; SLI $=$ specific language impairment.

dependent subject sample that applied the same image analysis methods examining volumetric asymmetry in language-association cortex in right-handed boys with autism and normal control boys. ${ }^{36}$ This prior study reported that inferior frontal cortex pars opercularis was larger in the right hemisphere in boys with autism and larger in the left hemisphere in normal control boys. In another study of subjects with autism, a decrease of gray matter was observed in Brodmann area 45 in the left hemisphere. ${ }^{35}$ The findings in inferior frontal cortex are consistent with previous studies indicating rightward asymmetry of the pars opercularis and triangularis of the inferior frontal gyrus in subjects with SLI. ${ }^{30,32,33,38}$

Further investigation is required to determine the developmental and behavioral causes and impact of the observed pattern of reversal of normal asymmetry in language-impaired subjects, both with or without autism. For example, in typically developing right-handed subjects, inferior frontal gyrus (Broca's area) tends to be larger in the left hemisphere, ${ }^{19,23-29}$ which suggests concordant left-hemisphere language as well as motor dominance. This is in contrast with the reported asymmetry in both autism language-impaired and SLI righthanded subjects, in whom the frontal language region is larger in the right hemisphere, suggesting that in these groups, language and motor dominance are segregated to opposite hemispheres. Based on the existing data, however, it is not possible to determine whether the development of language and motor dominance in opposing hemispheres causes complications or difficulties in normal language development, for example, or whether the abnormal cortical development is a compensatory sequelae of early language dysfunction.

This study suggests that exaggerated leftward asym- metry of the planum temporale is also more closely related to language diagnosis, because the two languageimpaired groups, ALI and SLI, both had similar and significant within-group PT asymmetry, whereas the two language-normal groups, ALN and NC, did not have significant PT asymmetry. PT was also significantly leftward asymmetric in a separate sample of boys with autism compared with developmentally normal boys in a prior study by our research team. ${ }^{36}$ Because the method of defining and measuring the structure volumes in the earlier study and this study are identical, we can directly compare data between these studies. Consistent with this study, the PT symmetry index in the earlier study was larger on the left in autism: $25 \%$ leftward asymmetry for the boys with autism in the prior study versus $27 \%$ larger PT on the left in ALI and SLI in this study. The main discrepancy between the PT asymmetry in the two studies is in the NC group (5\% leftward in prior study vs $16 \%$ leftward in this study). One possible explanation is related to agedifferences: the NC group in this study (10.4 years) was significantly older than the NC group in the earlier report (8.3 years). As suggested by several recent studies, it is possible that PT asymmetry increases with age in normal development. ${ }^{66,67}$

However, the pronounced leftward asymmetry of the planum temporale in the SLI and the autism groups is in contrast with findings from other studies. ${ }^{30,37} \mathrm{PT}$ measures may differ because of methodological issues such as surface measurements (vs volume measurements), the part of the PT measured, or the age of the subjects. ${ }^{28,68}$ In this study, PT volume includes both the horizontal and the posterior ascending part. More research is needed to clarify whether the differences in findings are attributable to method, age, or to subject variability (both within and between groups). 

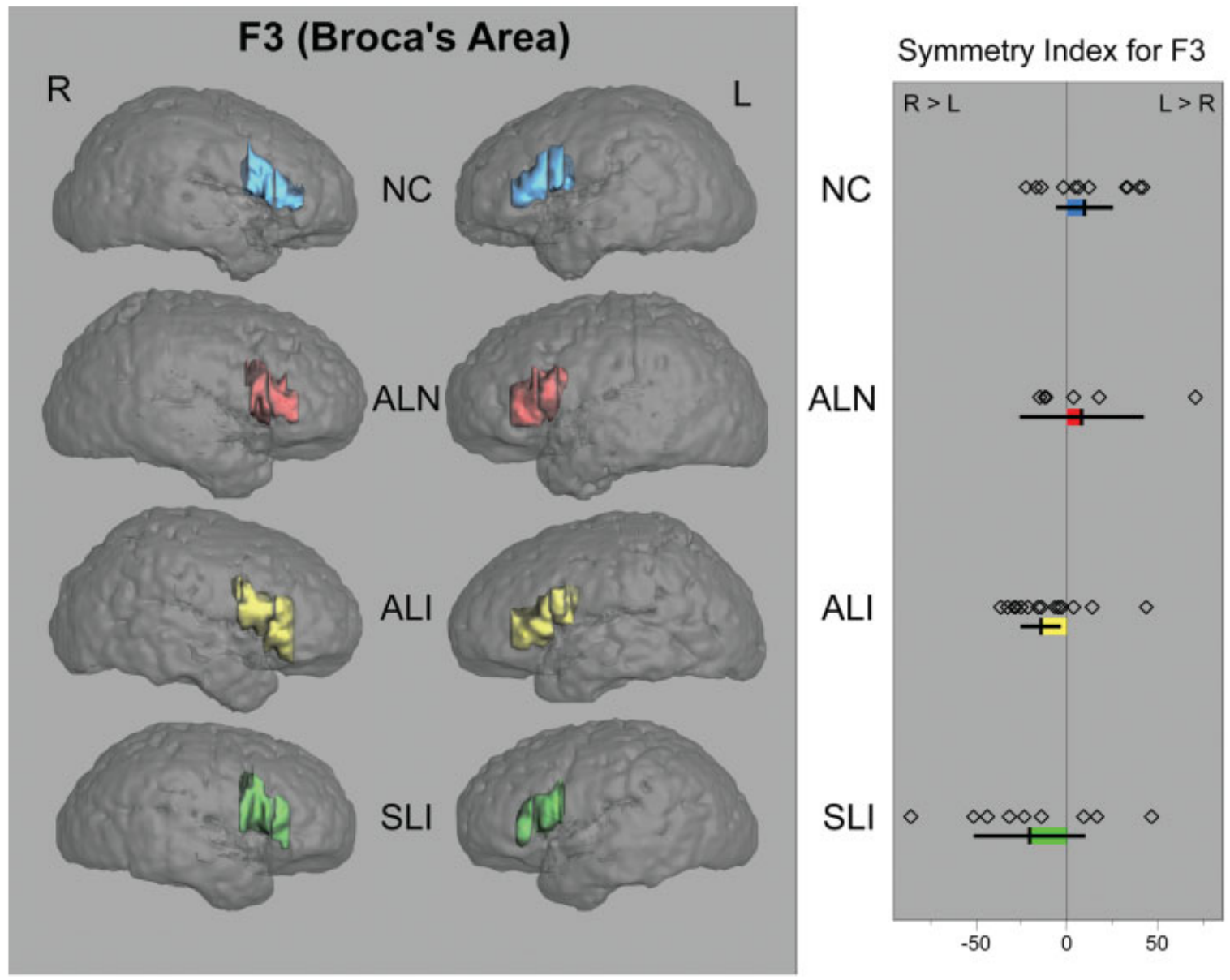

Fig 2. Surface renderings of four brain scans from subjects representative of each of the four study groups. Inferior frontal gyrus pars opercularis plus pars triangularis (Broca's area, "F3") is highlighted in each hemisphere. A scatterplot is shown by group of each subject's F3 asymmetry value. Group means, with color on bars indicating difference from zero, and error bars indicating $95 \%$ confidence intervals are shown below each group's data points. Positive symmetry index values indicate larger left-sided volumes. Subjects in the figure were chosen based on being closest to their respective group mean value of F3 asymmetry. The actual symmetry index for the cases shown (with group mean in parentheses) are NC, 11.2\% (9.7\%); ALN, 3.2 (8.2); ALI, - 14.7 (-14.2); and $S L I,-24.3$ (-20.3). $R=$ right; $L=$ left; $N C=$ normal control; $A L N=$ autism with normal language; $A L I=$ autism with language impairment; $S L I=$ specific language impairment.

In broader Broca's and Wernicke's area regions, including FO, PO, T1, and SG+AG, no differences were observed between groups in asymmetry measures. This is consistent with our earlier report and further supports the specificity of the asymmetry abnormality in F3 in language-impaired subjects with autism or SLI.

The reversal of frontal language cortex (Broca's area) asymmetry in language-impaired boys with autism and boys with SLI suggests similar deviations in cerebral cortical structure in language-association areas in these two disorders. In view of family studies and genetic linkage studies that point to overlapping gene regions, these findings support the hypothesis of a common neurobiological basis of language impairment in autism and SLI. One strength of this study was the decision to include only right-handed boys to limit potential confounding effects of handedness and gender, which are known to have an impact on language organization in the brain. A further strength is the effort to subdivide subjects with autism by language-impairment and compare these subgroups to control groups with and without languageimpairment. This allows us to begin to interpret our language-related asymmetry observations in terms of relationship to autism versus language-impairment domains. A weakness of this study is the small sample size in some of the groups, particularly language-normal autism subjects, that resulted from this subdivision of the autism group. A larger sample would be required to directly establish a difference between the subjects with autism with normal and impaired language skills. Future studies are needed to examine whether this result holds in male and female children and adults and whether there are correlations between other traits of autism and deviations in cortical structures. 
This work was supported by grants from the NIH (National Institute on Deafness and Other Communication Disorders, U19/PO1 DC 03610, H.T.-F., G.J.H.; National Institute of Mental Health, K08 MH01573-01, J.A.F.), and the National Alliance for Research on Schizophrenia and Depression (N.M.).

We thank E. Halpern for statistical consultation and S. McInerney for data representation.

\section{References}

1. APA. DSM-IV: Diagnostic and statistic manual of mental disorders. 4th ed. Washington, DC: American Psychiatric Press, 1994.

2. Lord C, Paul R. Language and communication in autism. In: Cohen DJ, Volkmar FR, eds. Handbook of autism and pervasive developmental disorders. 2nd ed. New York: Wiley, 1997.

3. Rapin I, ed. Preschool children with inadequate communication. Vol. 139. London: Mac Keith Press, 1996.

4. Tager-Flusberg H. Language impairments in children with complex neurodevelopmental disorders: the case of autism. In: Levy Y, Schaeffer JC, eds. Language competence across populations: toward a definition of specific language impairment. Mahwah, NJ: Lawrence Erlbaum Associates, 2003:297-321.

5. Tager-Flusberg H, Joseph RM. Identifying neurocognitive phenotypes in autism. Philos Trans R Soc Lond B Biol Sci 2003; 358:303-314.

6. Kjelgaard MM, Tager-Flusberg H. An investigation of language impairment in autism: implications for genetic subgroups. Lang Cogn Processes 2001;16:287-308.

7. Fisher SE, Lai CS, Monaco AP. Deciphering the genetic basis of speech and language disorders. Annu Rev Neurosci 2003;26: 57-80.

8. Santangelo SL, Folstein SE. Autism: a genetic perspective. In: Tager-Flusberg H, ed. Neurodevelopmental disorders. Cambridge, MA: MIT Press, 1999:431-447.

9. Fombonne E, Bolton P, Prior J, et al. A family study of autism: cognitive patterns and levels in parents and siblings. J Child Psychol Psychiatry 1997;38:667-683.

10. Folstein SE, Santangelo SL, Gilman SE, et al. Predictors of cognitive test patterns in autism families. J Child Psychol Psychiatry 1999;40:1117-1128.

11. Bailey A, Le Couteur A, Gottesman I, et al. Autism as a strongly genetic disorder: evidence from a British twin study. Psychol Med 1995;25:63-77.

12. Tomblin JB, Hafeman LL, O'Brien M. Autism and autism risk in siblings of children with specific language impairment. Int J Lang Commun Disord 2003;38:235-250.

13. IMGSAC. A full genome screen for autism with evidence for linkage to a region on chromosome 7q. International Molecular Genetic Study of Autism Consortium. Hum Mol Genet 1998; 7:571-578.

14. Barrett S, Beck JC, Bernier R, et al. An autosomal genomic screen for autism. Collaborative linkage study of autism. Am J Med Genet 1999;88:609-615.

15. O’Brien EK, Zhang X, Nishimura C, et al. Association of specific language impairment (SLI) to the region of $7 \mathrm{q} 31$. Am J Hum Genet 2003;72:1536-1543.

16. Bartlett CW, Flax JF, Logue MW, et al. A major susceptibility locus for specific language impairment is located on $13 \mathrm{q} 21$. Am J Hum Genet 2002;71:45-55.

17. Alarcon M, Cantor RM, Liu J, et al. Evidence for a language quantitative trait locus on chromosome $7 \mathrm{q}$ in multiplex autism families. Am J Hum Genet 2002;70:60-71.
18. Bradford Y, Haines J, Hutcheson H, et al. Incorporating language phenotypes strengthens evidence of linkage to autism. Am J Med Genet 2001;105:539-547.

19. Foundas AL. Language dominance and MRI asymmetries. Neurology 1995; 45:1635-1636.

20. Foundas AL, Leonard CM, Gilmore RL, et al. Pars triangularis asymmetry and language dominance. Proc Natl Acad Sci USA 1996;93:719-722.

21. Foundas AL, Leonard CM, Gilmore R, et al. Planum temporale asymmetry and language dominance. Neuropsychologia 1994; 32:1225-1231.

22. Moffat SD, Hampson E, Lee DH. Morphology of the planum temporale and corpus callosum in left handers with evidence of left and right hemisphere speech representation. Brain 1998; 121:2369-2379.

23. Falzi G, Perrone P, Vignolo LA. Right-left asymmetry in anterior speech region. Arch Neurol 1982;39:239-240.

24. Galaburda AM, Corsiglia J, Rosen GD, Sherman GF. Planum temporale asymmetry: reappraisal since Geschwind and Levitsky. Neuropsychologia 1987;25:853-868.

25. Kulynych JJ, Vladar K, Jones DW, Weinberger DR. Threedimensional surface rendering in MRI morphometry: a study of the planum temporale. J Comput Assist Tomogr 1993;17: $529-535$.

26. Foundas AL, Leonard CM, Heilman KM. Morphologic cerebral asymmetries and handedness. The pars triangularis and planum temporale. Arch Neurol 1995;52:501-508.

27. Foundas AL, Eure KF, Luevano LF, Weinberger DR. MRI asymmetries of Broca's area: the pars triangularis and pars opercularis. Brain Lang 1998;64:282-296.

28. Zetzsche T, Meisenzahl EM, Preuss UW, et al. In-vivo analysis of the human planum temporale (PT): does the definition of PT borders influence the results with regard to cerebral asymmetry and correlation with handedness? Psychiatry Res 2001; 107:99-115.

29. Watkins KE, Paus T, Lerch JP, et al. Structural asymmetries in the human brain: a voxel-based statistical analysis of $142 \mathrm{MRI}$ scans. Cereb Cortex 2001;11:868-877.

30. Gauger LM, Lombardino LJ, Leonard CM. Brain morphology in children with specific language impairment. J Speech Lang Hear Res 1997;40:1272-1284.

31. Lai CS, Fisher SE, Hurst JA, et al. A forkhead-domain gene is mutated in a severe speech and language disorder. Nature 2001; 413:519-523.

32. Watkins KE, Vargha-Khadem F, Ashburner J, et al. MRI analysis of an inherited speech and language disorder: structural brain abnormalities. Brain 2002;125:465-478.

33. Belton E, Salmond $\mathrm{CH}$, Watkins $\mathrm{KE}$, et al. Bilateral brain abnormalities associated with dominantly inherited verbal and orofacial dyspraxia. Hum Brain Mapp 2003;18:194-200.

34. Clark MM, Plante E. Morphology of the inferior frontal gyrus in developmentally language-disordered adults. Brain Lang 1998;61:288-303.

35. Abell F, Krams M, Ashburner J, et al. The neuroanatomy of autism: a voxel-based whole brain analysis of structural scans. Neuroreport 1999;10:1647-1651.

36. Herbert MR, Harris GJ, Adrien KT, et al. Abnormal asymmetry in language association cortex in autism. Ann Neurol 2002; 52:588-596.

37. Rojas DC, Bawn SD, Benkers TL, et al. Smaller left hemisphere planum temporale in adults with autistic disorder. Neurosci Lett 2002;328:237-240.

38. Plante E, Swisher L, Vance R, Rapcsak S. MRI findings in boys with specific language impairment. Brain Lang 1991;41: $52-66$. 
39. Salmond $\mathrm{CH}$, de Haan $\mathrm{M}$, Friston $\mathrm{KJ}$, et al. Investigating individual differences in brain abnormalities in autism. Philos Trans R Soc Lond B Biol Sci 2003;358:405-413.

40. Caplan D, Gow D, Makris N. Analysis of lesions by MRI in stroke patients with acoustic-phonetic processing deficits. Neurology 1995;45:293-298.

41. Caplan D, Hildebrandt N, Makris N. Location of lesions in stroke patients with deficits in syntactic processing in sentence comprehension. Brain 1996;119:933-949.

42. Oldfield RC. The assessment and analysis of handedness: the Edinburgh inventory. Neuropsychologia 1971;9:97-113.

43. Dean RS. Cerebral laterality and reading comprehension. Neuropsychologia 1978;16:633-636.

44. Dean RS. Assessing patterns of lateral preference. J Clin Neuropsychol 1982;4:124-128.

45. Elliot CD. Differential Ability Scales. San Antonio, TX: The Psychological Corporation, Harcourt Brace and Co., 1990.

46. Wechsler D. The Wechsler Intelligence Scale for Children. 3rd ed. San Antonio, TX: The Psychological Corporation, Harcourt Brace and Co., 1991.

47. Lord C, Rutter M, Le Couteur A. Autism Diagnostic Interview-Revised: a revised version of a diagnostic interview for caregivers of individuals with possible pervasive developmental disorders. J Autism Dev Disord 1994;24:659-685.

48. Lord C, Risi S, Lambrecht L, et al. The autism diagnostic observation schedule-generic: a standard measure of social and communication deficits associated with the spectrum of autism. J Autism Dev Disord 2000;30:205-223.

49. Semel E, Wiig EH, Secord WA. Clinical Evaluation of Language Fundamentals. 3rd ed. San Antonio, TX: The Psychological Corporation, Harcourt Brace and Co., 1995.

50. Korkman M, Kirk U, Kemp S. NEPSY: A Developmental Neuropsychological Assessment. San Antonio, TX: The Pyschological Corporation, Harcourt Brace and Co., 1998.

51. Conti-Ramsden G, Botting N. Classification of children with specific language impairment: longitudinal considerations. J Speech Lang Hearing Res 1999;42:1195-1204.

52. Slifer KJ, Cataldo MF, Cataldo MD, et al. Behavior analysis of motion control for pediatric neuroimaging. J Appl Behav Anal 1993;26:469-470.

53. Filipek PA, Kennedy DN, Caviness VS Jr. A method of morphometric analysis of the human brain based upon magnetic resonance images. [abstract] Ann Neurol 1988;24:356. [Abstract 177]

54. Filipek PA, Richelme C, Kennedy DN, Caviness VS Jr. The young adult human brain: an MRI-based morphometric analysis. Cerebr Cort 1994;4:344-360.
55. McGraw KO, Wong SP. Forming inferences about some intraclass correlation coefficients. Psychol Methods 1996;1:30-46.

56. Shrout PE, Fleiss JL. Intraclass correlations: uses in assessing rater reliability. Psychol Bull 1979;86:420-428.

57. Woods RP, Grafton ST, Holmes CJ, et al. Automated image registration: I. General methods and intrasubject, intramodality validation. J Comput Assist Tomogr 1998;22:139-152.

58. Woods RP, Grafton ST, Watson JD, et al. Automated image registration. II. Intersubject validation of linear and nonlinear models. J Comput Assist Tomogr 1998;22:153-165.

59. Worth AJ, Makris N, Meyer JW, et al. Semiautomatic segmentation of brain exterior in magnetic resonance images driven by empirical procedures and anatomical knowledge. Med Image Anal 1998;2:315-324.

60. Talairach J, Tournoux P. Co-planar stereotaxic atlas of the human Brain. New York: Thieme, 1988.

61. Worth AJ, Makris N, Patti MR, et al. Precise segmentation of the lateral ventricles and caudate nucleus in MR brain images using anatomically driven histograms. IEEE Trans Med Imaging 1998;17:303-310.

62. Arnold JB, Liow JS, Schaper KA, et al. Qualitative and quantitative evaluation of six algorithms for correcting intensity nonuniformity effects. Neuroimage 2001;13:931-943.

63. Rademacher J, Galaburda AM, Kennedy DN, et al. Human cerebral cortex: localization, parcellation and morphometry with magnetic resonance imaging. J Cogn Neurosci 1992;4:352-374.

64. Caviness VS Jr, Meyer JW, Makris N, Kennedy DN. MRIbased topographic parcellation of the human neocortex: an anatomically specified method with estimate of reliability. J Cogn Neurosci 1996;8:566-587.

65. Kramer CY. Extension of multiple range tests to group means with unequal numbers of replications. Biometrics 1956;12: 301-310.

66. Amunts K, Schleicher A, Ditterich A, Zilles K. Broca's region: cytoarchitectonic asymmetry and developmental changes. J Comp Neurol 2003;465:72-89.

67. Sowell ER, Thompson PM, Rex D, et al. Mapping sulcal pattern asymmetry and local cortical surface gray matter distribution in vivo: maturation in perisylvian cortices. Cereb Cortex 2002;12:17-26.

68. Shapleske J, Rossell SL, Woodruff PW, David AS. The planum temporale: a systematic, quantitative review of its structural, functional and clinical significance. Brain Res Brain Res Rev 1999;29:26-49. 